\title{
Aplicación "UDAFORMIN" para la determinación del tipo de fortificación minera
}

DOI: $10.46932 / \mathrm{sfjdv2n1-080}$

Received in: November 1st, 2020

Accepted in: December 30th, 2020

\section{RESUMEN}

Patricio Feijoo Calle

Escuela de Ingeniería en Minas

Facultad de Ciencia y Tecnología

Universidad del Azuay (Ecuador)

E-mail: pfeijoo@uazuay.edu.ec

\section{Adrián Bravo León}

Escuela de Ingeniería en Minas

Facultad de Ciencia y Tecnología

Universidad del Azuay (Ecuador)

E-mail:pfeijoo@uazuay.edu.ec

Nicolás Escandón Calle

Escuela de Ingeniería en Minas

Facultad de Ciencia y Tecnología

Universidad del Azuay (Ecuador)

E-mail: pfeijoo@uazuay.edu.ec

En minería, como túneles y taludes, para la evaluación del tipo de fortificación, los parámetros importantes que se deben introducir, para el cálculo de la misma, deben pasar por un proceso de análisis, que muchas veces puede ocasionar, por la falta de experiencia, errores de apreciación y aproximación. Esta caracterización es de importancia para la seguridad de los trabajadores. Por esta razón se vio importante preparar una aplicación en Visual Basic, la cual permita, solamente con la introducción de los parámetros iniciales, como la resistencia a la compresión, Rock Quality Designation (RQD), bloque típico, condiciones de fracturas, agua subterránea, etc. y en función de la programación establecida, encontrar resultados de Rock Mass Rating (RMR), Calidad de la Roca (Índice Q), Tiempo de Auto sostenimiento y Tipo de Fortificación necesaria.

Palabras clave: minería, visual, Basic, fortificación.

\section{INTRODUCCIÓN}

Para el desarrollo de actividades mineras, la estabilidad y sostenimiento de galerías o taludes es una parte fundamental y se establece como una de las labores más importantes, puesto que, la fortificación correcta cumple con su objetivo de evitar derrumbes o caída de bloques y en consecuencia, garantiza la seguridad, principalmente del personal, de las herramientas o equipos y la producción, por lo que de esta manera, con una fortificación adecuada y técnica se logrará evitar accidentes laborales. Por lo expuesto podemos generar, para el personal, una zona y ambiente de trabajo seguros, logrando que su desempeño sea mayor y proporcionando seguridad para la empresa. 
Ahora bien, para determinar el tipo de fortificación se realiza una serie de cálculos y análisis de las clasificaciones geo mecánicas, y muchas veces estas operaciones pueden ser erradas o mal aproximadas, a más de que estos procesos de cálculo pueden ser tediosos y en muchas ocasiones generan confusión y/o errores de apreciación o visualización.

Es así, que hemos encontrado la necesidad de implementar la tecnología, para poder principalmente determinar o elegir el tipo de fortificación apropiada, en los trabajos en minería. Con esta aplicación estamos en la capacidad de determinar inicialmente valores del Rock Mass Rating (RMR) e Índice Q, el tiempo de auto sostenimiento del macizo rocoso, factor de seguridad y finalmente establecer el tipo específico de fortificación a utilizarse en la labor minera, ejecutando los cálculos respectivos de manera confiable, por medio de la aplicación de diferentes métodos y comparaciones.

Esta aplicación se la ha desarrollado en Visual Basic, y la hemos denominado UDAFORMIN. Es una herramienta de ágil manejo, permite un ahorro considerable en tiempo y de una manera más segura nos presenta los tipos de fortificación ideales para la obra estudiada.

\section{METODOLOGIA}

La base fundamental de este trabajo está en la Mecánica de Rocas, la que resuelve entre otros los problemas del comportamiento de la matriz rocosa y del comportamiento de la roca in situ con las discontinuidades inherentes (macizo rocoso) [1].

Debido a lo descrito se llegará a definir la fortificación o estabilización de la obra que se está analizando. La fortificación es el conjunto de procedimientos que permiten mantener estables las labores cuando su condición no es auto soportante en una mina subterránea [2].

En minería subterránea la fortificación debe ser confiable, desde el inicio de las labores mineras subterráneas, para de esta manera garantizar un lugar de trabajo seguro.

La calidad del proyecto depende de la confiabilidad de las investigaciones de campo y laboratorio, así como la capacidad del proyectista para interpretar los resultados experimentales, definir los parámetros del proyecto y, principalmente, analizar los escenarios posibles que pueden alterar las condiciones de resistencia al corte o reducir los factores de seguridad [3].

Para la elección del tipo de sostenimiento o fortificación a utilizar en una obra subterránea, existen una serie datos o valores iniciales que se deben obtener en campo y con experticia del caso. Hay varios criterios que se deben considerar para elegir el tipo de fortificación adecuada que se empleará en un lapso de tiempo, o de forma permanente [4].

Los criterios que se deben analizar son:

- Condiciones mecánicas y geo estructurales, existentes en el macizo. 
- Forma y dimensiones de la sección transversal de la excavación.

- Su vida de servicio y uso.

- Grado de estabilidad del sistema macizo-excavación.

- Presencia de agua y sus características.

Determinadas estas características, se procede al desarrollo de la metodología para la determinación del tipo de fortificación.

Siendo fundamental aplicar las clasificaciones denominadas RMR e Índice Q, para lo cual se ha desarrollado, en el lenguaje de programación de Microsoft, Visual Basic.NET, el cual es parte de la nueva generación del lenguaje ya conocido Visual Basic.

Está diseñado para la creación de aplicaciones orientadas a objetos brindando productividad y seguridad. Los destinos más comunes de este lenguaje son dispositivos móviles, web y computadoras con el sistema operativo Windows. Las aplicaciones construidas con Visual Basic se generan con los servicios de Common Language Runtime y todos los lenguajes que tienen como destino Microsoft .NET Framework, se benefician de la seguridad ampliada, interoperabilidad entre lenguajes, recolección de elementos no utilizados y compatibilidad de versiones mejoradas [5].

Establecida esta aplicación (figura 1), la misma presenta una serie de ventanas, iniciando por el ingreso de datos, lo cual podemos ver en la figura 2 :

Fig. 1. Ventana para inicio de la aplicación UDAFORMIN

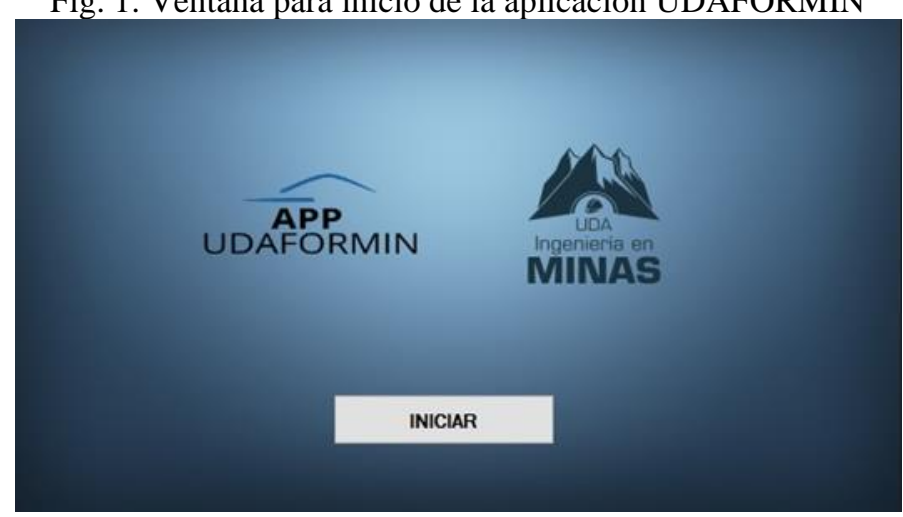


Fig. 2. Ventana para introducción de datos iniciales

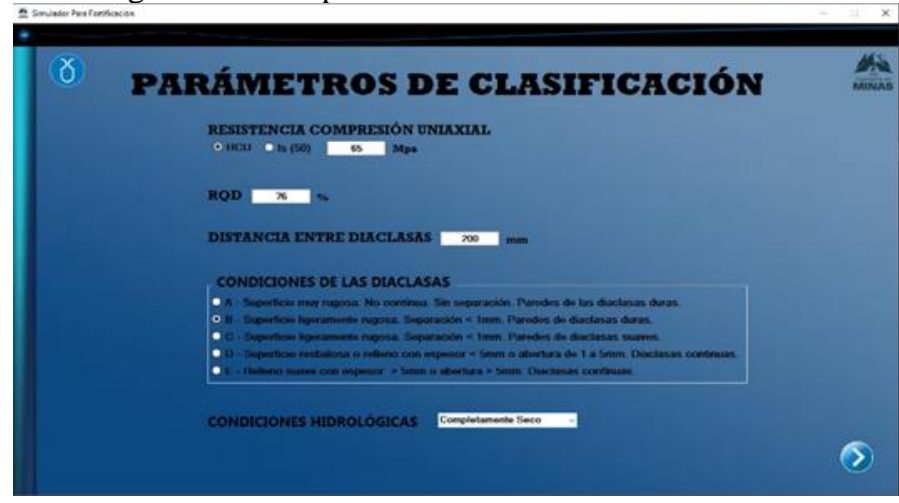

La aplicación, como se mencionó anteriormente, se desarrolló con el lenguaje de programación Visual Basic. El desarrollo del código se inició identificando los problemas iniciales para el cálculo. La aplicación requiere de ciertos datos que son ingresados manualmente por el usuario como son: Resistencia a la Compresión Uniaxial (RCU) o Índice de Point Load Test (Is(50)), RQD, separación y condiciones de las diaclasas y condiciones hidrológicas. A cada uno de estos valores se les debe asignar un respectivo puntaje, que es calculado mediante ecuaciones. Estas ecuaciones son codificadas mediante órdenes y procedimientos correctamente establecidos por el desarrollador, para el entendimiento del ordenador, y así este pueda asignar automáticamente el puntaje correcto. Luego se declaró un código para que la aplicación asigne automáticamente un puntaje de corrección y obtener como resultado el puntaje de RMR. (Figura 3).

Fig. 3. Pantalla con el resultado de RMR y Clase de Roca

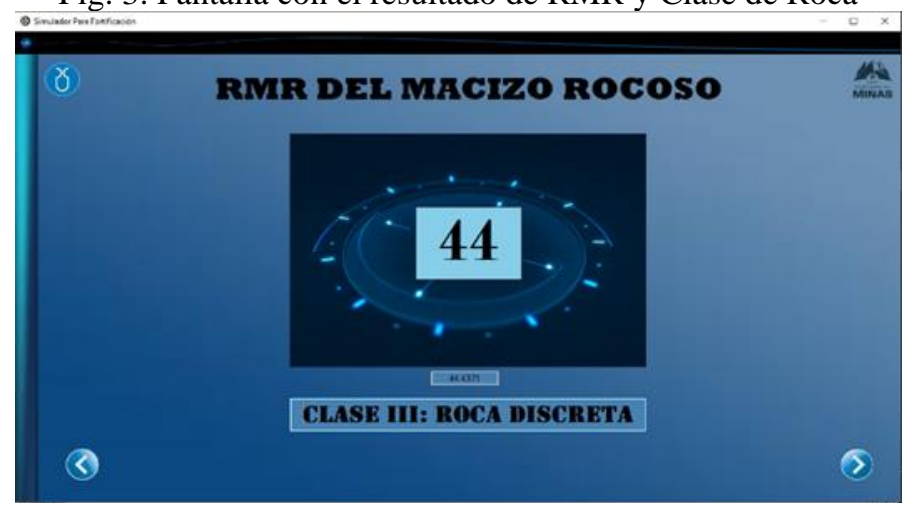

El valor de la luz del túnel es otro dato que el usuario debe ingresar manualmente, para el cálculo del factor de seguridad y el tiempo de auto sostenimiento. Se debe cumplir órdenes, reglas y variables declaradas en el lenguaje de programación para que la aplicación pueda asignar y mostrar resultados correctos. Los últimos datos que el usuario debe ingresar manualmente servirán para el cálculo del índice Q, que, por medio de una fórmula que correctamente codificada en el lenguaje, la aplicación calculará y mostrará el valor del Índice Q. Finalmente el usuario deberá seleccionar el tipo de excavación que se 
realizará y el programa evaluará de manera automática, obedeciendo las especificaciones ingresadas por el desarrollador, cuál será el soporte adecuado que se deberá utilizar, esto se puede ver en las figuras 4,5 , 6 y 7.

Si los resultados obtenidos no coinciden con las especificaciones establecidas en el código, el programa mostrará un mensaje distinto. Todos los resultados obtenidos: RMR, factor de seguridad, tiempo de auto sostenimiento, Índice Q y el tipo de fortificación, serán exteriorizados en un documento en Microsoft Word.

Fig. 4. Pantalla con el resultado del Factor de Seguridad

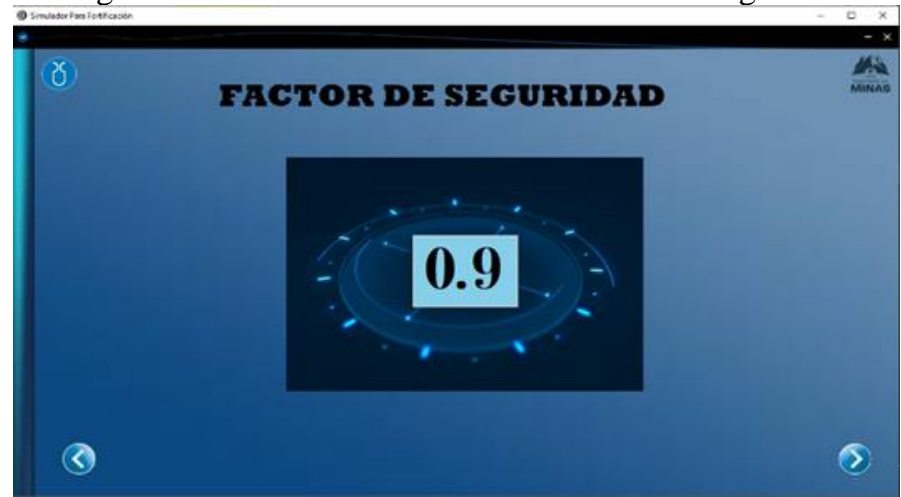

Fig. 5. Pantalla con el resultado del Tiempo de Auto sostenimiento

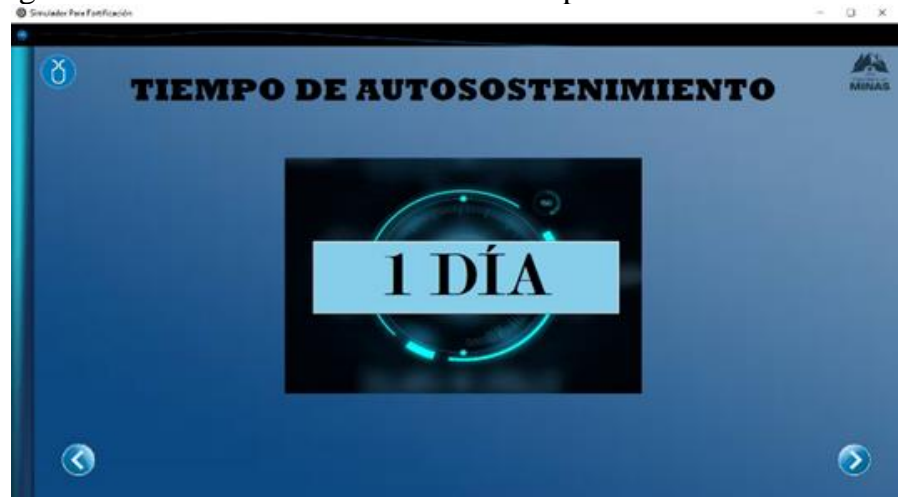

Fig. 6. Pantalla con el resultado del Índice Q

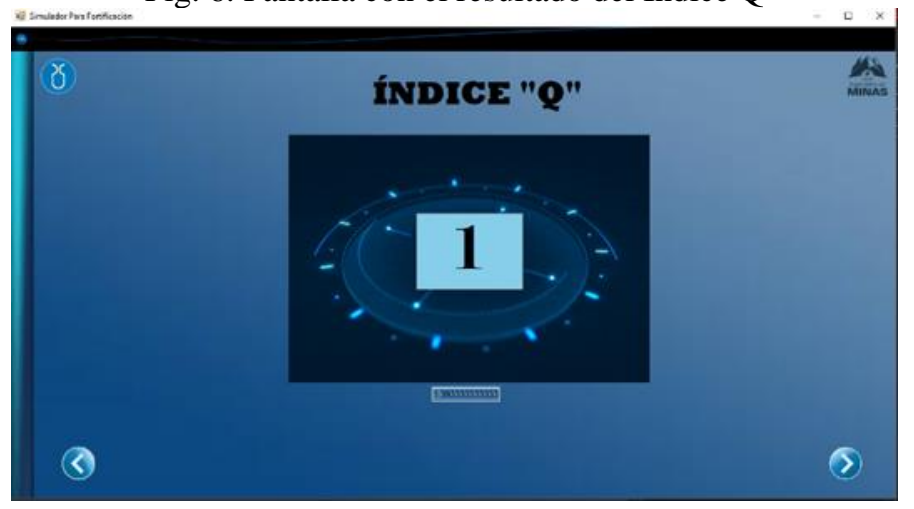


Fig. 7. Pantalla con el resultado del Soporte Recomendado

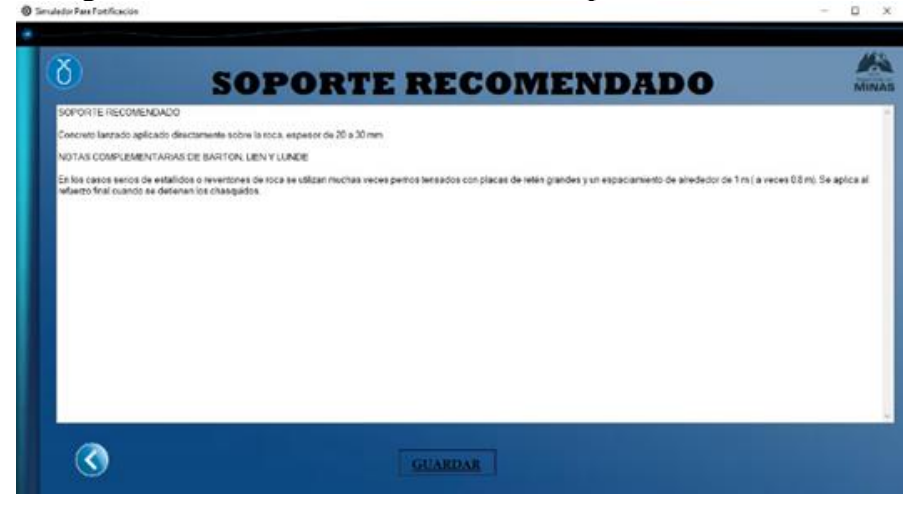

\section{RESULTADOS Y COMPARACIÓN}

Luego de ejecutada la fase de programación de la aplicación, se verificó su funcionamiento mediante la obtención de resultados con el ingreso de datos de estudios anteriores, con la intención de corroborar si la aplicación presenta similitud con los resultados de otras investigaciones o trabajos. Para esto se han tomado como referencia los siguientes estudios, los cuales se pueden encontrar en la web:

1. Correlación entre el índice RMR de Bieniawski y el Índice Q de Barton en formaciones sedimentarias de grano fino, presente en:

http://informesdelaconstruccion.revistas.csic.es/index.php/informesdelaconstruccion/artic le/view/5854/6841

De este estudio obtuvimos los datos iniciales, los cuales son:

\begin{tabular}{|l|l|l|l|l|l|}
\hline $\begin{array}{l}\text { RCU } \\
(\mathrm{MPa})\end{array}$ & $\begin{array}{l}\text { RQD } \\
(\%)\end{array}$ & $\begin{array}{l}\text { Distancia de } \\
\text { las diaclasas } \\
(\mathrm{mm})\end{array}$ & $\begin{array}{l}\text { Condiciones de } \\
\text { las diaclasas }\end{array}$ & $\begin{array}{l}\text { Condiciones } \\
\text { Hidrológicas }\end{array}$ & LUZ $(\mathrm{m})$ \\
\hline 31.89 & 76 & 200 & $\begin{array}{l}\text { Superficie } \\
\text { ligeramente } \\
\text { rugosa. } \\
\text { Separación } \\
<1 \text { mm. Paredes } \\
\text { diaclasas duras }\end{array}$ & $\begin{array}{l}\text { Completamente } \\
\text { Seco }\end{array}$ & 6 \\
\hline
\end{tabular}

\begin{tabular}{|l|l|l|l|l|l|}
\hline Jn & Jr & Ja & JW & SRF & Tipo de excavación \\
\hline 9 & 1.5 & 6 & 0.66 & 1 & $\begin{array}{l}\text { Excavaciones mineras permanentes. Túneles de } \\
\text { conducción de agua para obras hidroeléctricas } \\
\text { túneles piloto, excavaciones parciales para } \\
\text { cámaras subterráneas grandes. }\end{array}$ \\
\hline
\end{tabular}

Los resultados del estudio son:

\begin{tabular}{|l|l|}
\hline RMR = 63 & INDICE Q $=1.39$ \\
\hline
\end{tabular}


y los resultados de la aplicación UDAFORMIN:

$$
\text { RMR }=62.579 \quad \text { INDICE Q }=1.39333
$$

Además de lo expuesto la aplicación UDAFORMIN nos entrega la siguiente información adicional:

- Fortificación recomendada por la aplicación:

concreto lanzado aplicado directamente sobre la roca espesor de 20 a 30 m.

- Notas complementarias de Barton, Lien y Lunde:

En los casos serios de estallidos o reventones de roca se utilizan muchas veces pernos tensados con placas de reten grandes y un espaciamiento alrededor de $1 \mathrm{~m}$ (a veces de $0.8 \mathrm{~m}$ ).

Se aplica al refuerzo final cuando se detienen los chasquidos.

2. Sectorización Geotécnica del Túnel de Drenaje Etapa 2, expansión Norte de mina Sur, división CODELCO Norte, en:

http://www.tesis.uchile.cl/tesis/uchile/2007/rayo_c/sources/rayo_c.pdf

De este estudio obtuvimos los datos iniciales, los cuales son:

\begin{tabular}{|l|l|l|l|l|l|}
\hline $\begin{array}{l}\text { RCU } \\
(\mathrm{MPa})\end{array}$ & $\begin{array}{l}\text { RQD } \\
(\%)\end{array}$ & $\begin{array}{l}\text { Distancia de } \\
\text { las diaclasas } \\
(\mathrm{mm})\end{array}$ & $\begin{array}{l}\text { Condiciones } \\
\text { de las } \\
\text { diaclasas }\end{array}$ & $\begin{array}{l}\text { Condiciones } \\
\text { Hidrológicas }\end{array}$ & LUZ (m) \\
\hline $20-80$ & $65-80$ & 2 a 7 & $\begin{array}{l}\text { Onduladas } \\
\text { lisas } \\
\text { onduladas } \\
\text { rugosas }\end{array}$ & $\begin{array}{l}\text { Seca o presenta } \\
\text { flujos de agua con } \\
\text { caudales menores a } \\
5 \text { lt/min }\end{array}$ & 4.5 \\
\hline
\end{tabular}

\begin{tabular}{|l|l|l|l|l|l|}
\hline Jn & Jr & Ja & Jw & SRF & Tipo de excavación \\
\hline $6-12$ & $2-3$ & 4 & 1 & 2.5 & $\begin{array}{l}\text { Excavación subterránea, } \\
\text { el Túnel de Drenaje. }\end{array}$ \\
\hline
\end{tabular}

Los resultados del estudio son:

\begin{tabular}{|l|l|}
\hline RMR $=35$ a 65 & INDICE $Q=1.1$ a 1.8 \\
\hline
\end{tabular}


y los resultados de la aplicación UDAFORMIN:

\begin{tabular}{|l|l|l|}
\hline RMR & INDICE Q \\
59.6054 & 1.625 & \\
\hline
\end{tabular}

\section{CONCLUSIONES}

La aplicación UDAFORMIN puede ser una herramienta de gran ahorro en tiempo y evita errores de visualización y cuantificación en la determinación de RMR o Índice Q, lo que garantiza que los resultados puedan ser aceptados para tranquilidad de los trabajadores y de la obra en construcción y desarrollo.

Como se puede observar en las figuras 1 a 7 la aplicación UDAFORMIN es muy accesible y amigable para su manejo. Lo que se debe tener presente es que los datos iniciales ingresados deben tener la certeza y confiabilidad del ingeniero o experto que los determine en campo, esto debido a que la aplicación no genera ningún tipo de corrección de los valores ingresados.

Podemos registrar, que en los dos estudios tomados en consideración para la comparación con la aplicación UDAFORMIN, los resultados están en orden de similitud bastante aproximada, lo que hace prever que el desarrollo programático de la aplicación es correcto y confiable.

Una vez evaluados los resultados, que se han obtenido en las experiencias llevadas a cabo en estos dos ejemplos y en varios que en el presente estudio no se incluyen por espacio, reconocemos el empleo de la aplicación UDAFORMIN para la determinación de la fortificación recomendada para cada caso de estudio.

Esta aplicación es necesaria en el caso de minería para el estudio geotécnico y comportamiento de la roca y macizo rocoso; pues en la práctica se ha demostrado que existen otras variables a tomar en consideración. La profundización en el conocimiento de los diferentes materiales rocosos, la corroboración en el tiempo en la eficiencia de la aplicación, son puntos que deben ser tratados para que UDAFORMIN se ponga a punto y sea avalada. 


\section{REFERENCIAS BIBLIOGRÁFICAS}

[1] Galván, M. (2015), Mecánica de Rocas: Correlación entre la Resistencia a Carga Puntual y la Resistencia a Compresión Simple, Colombia, p. 23.

[2] Gonzáles, R. (2013), Guía N 5 de operación para la Pequeña Minería Fortificación y Acuñadura. Chile, p. 4.

[3] Gerscovich, D. (2015), Estabilidad de Taludes. Colombia, p. 33-34.

[4] Sari, J., y Rodas, D. (2013), Propuesta del Sistema de Fortificación que se debe emplear a lo largo de la veta F-10 de la mina subterránea Liga de Oro de la empresa SOMILOR S.A., Trabajo de Graduación, Universidad de Cuenca, Ecuador.

[5] Dollard, K., y Cai, S. (2018), https://docs.microsoft.com/es-es/dotnet/visual-basic/ Microsoft. 